\title{
Ways to transform your healing environment
}

\section{Editorial}

For my first editorial I will return to the foundation of nursing using precepts by Florence Nightingale. What would Florence do (WWFD) concerning transformation of the unit or facility environment where we work as nurses? The specialty unit for this application of precepts will be a medical oncology unit.

Florence was well grounded on the environmental influences on patients, families and staff and also the need to manipulate that environment to promote or put the patient in the best experience for healing. Environmental factors that Florence promoted were fresh air, clean water, nutritious food, sunlight, clean environment, and sanitary disposal of wastes. ${ }^{1}$

Fresh air is not easy to procure on a unit on the upper floor of an inner city facility. The pumping in of heppa filtered, fresh air would be advantageous to people with lung diseases or symptoms. It would be refreshing for staff and visitors alike because it would promote oxygenation. Next, clean water can be promoted by filtering drinking water at the source of the water and ice dispenser on the unit. Any drinking water fountains throughout the facility should also be filtered and able to fill water bottles.

Nutritious food should be available for all. Patients on my unit are also Immuno compromised due to cancer diagnosis and treatments. Nutritious food would be supportive of the oncology patient's immune function. Cancer treatments also can decrease the appetite and ability to taste, and increase nausea and vomiting. Flexible meal choices and nutrient rich foods are essential to this patient population. The ability of the staff to access nutritious foods during the evening and night shifts should be available and promoted as well.

Sunlight is important to decrease seasonal depressive disorders, decrease sleepiness during daytime, and promote vitamin d production and to promote warmth. Promoting the ability of the patients, families and staff to be in a room with a window that accesses sunlight is crucial. Earlier hospitals had solariums with windowed sides and ceiling. The staff would bring their lunch to this area to eat or take a break. Patients could be wheeled or walk to this room to sit and look out over the city. It was a healing environment.

Cleanliness is another area that Florence promoted. She would approve of non-toxic cleansing agents used in healthcare. Tea tree oil is a gentler, more natural cleanser to clean isolation rooms for patients
Volume 3 Issue 3 - 2017

\author{
Penny Kessler \\ Department of Nursing, University of Minnesota, USA
}

Correspondence: Penny Kessler, Department of School of Nursing, University of Minnesota, USA, Email kess1056@umn.edu

Received: July 8, 2017 | Published: July 17, 2017

with Methicillin resistant Staphylococcus aureus (MRSA). Cleansers currently used are sometimes noxious and not helpful to patients with nausea and vomiting from treatments. ${ }^{2}$ Next, changes with the disposal of wastes are needed. Recycling should be promoted as well as using "reusable" equipment when possible and cutting down on "single use" supplies as much as possible.

Lastly, Florence would study the unit environment, such as we are doing in this editorial, and would make observations and note occurrences of optimal and less than optimal healing environmental experiences. She would then take these observations to the people who could make changes (patient advocacy groups, families involved in improving healthcare, staff and management). In this way she would influence the changes needed to create an optimal healing environment for patients. Florence would have liked the opportunities to study and try to improve the healthcare experience. Take awayhow can you transform your work environment to promote optimal healing?

\section{Acknowledgements}

None.

\section{Conflict of interest}

The author declares no conflict of interest.

\section{References}

1. Nightingale F. Notes on Nursing: What it is and what it is not. New York, USA: Appleton \& Company; 1860.

2. Mayo. Tea tree oil; 2013. 